\title{
A single-step identification strategy for the coupled TITO process using fractional calculus
}

Transactions of the Institute of Measurement and Control $|-| \mid$

\author{
Kajal Kothari, Utkal Mehta $\unrhd$ and Ravneel Prasad
}

\begin{abstract}
The reliable performance of a complete control system depends on accurate model information being used to represent each subsystem. The identification and modelling of multivariable systems are complex and challenging due to cross-coupling. Such a system may require multiple steps and decentralized testing to obtain full system models effectively. In this paper, a direct identification strategy is proposed for the coupled two-input two-output (TITO) system with measurable input-output signals. A well-known closed-loop relay test is utilized to generate a set of inputs-outputs data from a single run. Based on the collected data, four individual fractional-order transfer functions, two for main paths and two for cross-paths, are estimated from single-run test signals. The orthogonal series-based algebraic approach is adopted, namely the Haar wavelet operational matrix, to handle the fractional derivatives of the signal in a simple manner. A single-step strategy yields faster identification with accurate estimation. The simulation and experimental studies depict the efficiency and applicability of the proposed identification technique. The demonstrated results on the twin rotor multiple-input multiple-output (MIMO) system (TRMS) clearly reveal that the presented idea works well with the highly coupled system even in the presence of measurement noise.
\end{abstract}

\section{Keywords}

Fractional-order models, TITO process, Haar wavelet, time delay, twin rotor, relay feedback

\section{Introduction}

In industry, various sophisticated processes have a multivariable nature. These processes are complex, owing to multiple inputs, multiple outputs and the loop interactions that make the identification of the multiple-input multiple-output (MIMO) process difficult and time-consuming. Due to strong interactions between outputs and inputs, such processes are more complex to study than single-input single output (SISO) systems. A closed-loop identification strategy is preferred to extract the process dynamics when processes are not suitable for open-loop configuration. These processes cannot be tested in the open-loop configuration due to instability, safety and reliability issues. In this work, the most general subcategory of the MIMO process, the two-input two-output (TITO) process, has been considered, with particularly emphasis on fractional process identification. The helicopter is the most familiar example of such a complex system with rotary wings.

Nowadays, more system models have adopted a noninteger (fractional-order) transfer function for system dynamics representation. An application of fractional calculus (FC) has gained immense popularity due to its superior accuracy and an additional degree of freedom. This is the reason for the increasing application of FC in the field of control engineering (Chen et al., 2009; Gutiérrez et al., 2010; Monje et al., 2010; Petráš, 2011). A detailed review on fractional-order (FO) system identification was represented by Kothari et al. (2019). In the literature, various operational matrix-based identification methods have been developed using fractional approaches for SISO systems (Kothari et al., 2018, 2020; Li et al., 2015; Tang et al., 2017). However, the FO operational matrix approach-based method is not available for TITO processes in the literature.

Various identification techniques have been reported in the literature for multivariable processes based on integer low-order models. Generally, the processes were modelled as a pair of first-order integer transfer functions for TITO dynamics. The step-test-based identification was firstly illustrated by Wang et al. (2000), but applicable with decouplers. A relay feedback technique and variances in the relay with the type of tests are shown in studies (Bajarangbali and Majhi, 2012; Berner et al., 2017a, 2017b; Kalpana et al., 2015; Mehta and Majhi, 2011; Nema and Padhy, 2015; Semino and Scali, 1998). The improved frequency-domain identification technique for MIMO processes was described first by Semino and Scali (1998), who utilized a two-step strategy. In the same way, the relay feedback time domain explicit equations considering the controller in the loop have been adopted for

Electrical and Electronics Engineering, The University of the South Pacific, Fiji

\section{Corresponding author:}

Utkal Mehta, Electrical and Electronics Engineering, The University of the South Pacific, Laucala Campus, Suva, 3232337, Fiji

Email: utkal.mehta@usp.ac.fj 
cascade process identification (Mehta and Majhi, 2011). A similar approach was used to revisit TITO processes (Bajarangbali and Majhi, 2012). Further, Kalpana et al. (2015) developed an identification scheme for the non-square MIMO system using time-domain relay response expressions. The expressions were derived using the block-relative-gain method and were verified on the two-tank cylindrical system. A state-space-based time-domain strategy for the TITO process was illustrated to identify first-order models by Nema and Padhy (2015). In this method, the conventional relay was utilized without a controller. Berner et al. (2017a) demonstrated autotuner TITO process identification based on a relay method. This method uses only a single relay test to identify four individual transfer functions, and one does not need to wait for convergence. Therefore, it makes identification faster. However, this method did not use controllers in loops. Authors have evaluated practically a relay autotuner with short excitation on a quadruple-tank setup (Berner et al., 2017b). Besides the relay test, some alternative techniques have been reported to identify TITO processes. For instance, the applied source separation method (Broman et al., 1999), curve fitting and genetic algorithm-based method (Viswanathan et al., 2001), a closed-loop reaction curve method (Ram and Chidambaram, 2014) and nonlinear least square minimization method (Ram and Chidambaram, 2016) have been well explained in the literature. Various techniques using step response data were developed for parameter estimations and include a sequence of step signals ( $\mathrm{Li}$ et al., 2005a, 2005b; Pereira et al., 2017). A step response test with particle swarm optimization and search algorithm for the multivariable process was presented by Jin et al. (2012). Even though much has been discussed for TITO processes, very few studies have been discussed for FO identification applied for TITO or multivariable processes. Recently, a decoupled method on FO-TITO processes (Li and Chen, 2014) and a multivariable FO model with time delay (San-Millan et al., 2017) have clearly shown superior performance. This study was carried out to demonstrate how non-integer models would help to understand complex processes accurately.

In summary, it is clear that most of the methods available require two-step procedures and estimate classical low-order models. Among them, some methods require decoupling before identification. Also, many methods utilize conventional relays without controllers. Such methods are only applicable to stable systems. In the case of an unstable system with a large delay it fails due to the absence of controllers. The Smith predictor-based sliding mode controller was discussed to deal with large delayed unstable plant (Mehta and Rojas, 2017). Also, an FO sliding mode controller was applied to the problem of trajectory control of a ball in a ball-and-plate system by Roy et al. (2018). Although some good methods utilized the closed-loop identification strategy, they are very complex. The single-step method developed by Berner et al. (2017a) is a fast open-loop identification strategy. However, it was developed with a conventional relay. The above limitations encourage researchers to develop a direct identification strategy using simple closed-loop data. The data can be easily obtained after a single test using relay autotuning. Such a method shall be flexible enough to consider simple step response data for identification. Overall, this work uses a preload relay to obtain benefits, such as improved stability during identification, quality estimation, shorter test time and simplify implementation. More importantly, it is desired to complete the test in a single run to estimate all four processes, including loop interaction.

When an arbitrary system is unstable because of too many inputs and outputs, it necessitates a promising identification algorithm to estimate the main paths and loop interactions accurately. Owing to the complex nature of highly coupled TITO processes and FC, how to deal with identification problems in FO with less complexity and better accuracy is an open problem. In this article, the TITO process is considered with no prior knowledge of its counterpart integer-order model or structure. The work is aimed at coupled processes that do not take numerous tests and do not only depend on a single ultimate frequency point. This paper proposes a singlestep identification strategy based on Haar wavelet and relay feedback. The presented method uses a well-adopted relay feedback test to generate input-output responses in a closed loop and relay with a controller inducing a sustained oscillation to stimulate the TITO process. The technique can estimate the process parameters by performing a single-step closed-loop relay experiment without decentralizing the process. Four independent fractional order models (FOMs) for the considered TITO processes are obtained using simple measurable responses. The performance of the model is illustrated with experimental validation on a twin rotor MIMO system (TRMS). The strategy of linear identification is developed for a cross-coupled TRMS setup in a single step using FO double-pole models. A fractional differential equation can increase the computational complexity due to the noninteger order. However, the proposed method converts the complex integro-differential equations of any orders into simple algebraic equations. It can estimate four independent FOMs without additional effort to decouple the process and also without filtering the noisy measured data. Finally, this new technique is verified on relevant process model examples.

\section{Fractional derivative and integral concepts}

A non-integer or FO derivative and integral can be explained by its operator in a basic form as

$$
{ }_{c} \mathscr{D}_{t}^{\lambda}=\left(\frac{d^{\lambda}}{\mathrm{dt}^{\lambda}}\right)
$$

where $c$ and $t$ are the bounds of the operation and $\lambda(\lambda \in R)$ is the real order whose value depicts the nature of the operation. Usually, a positive value of $\lambda$ exhibits fractional differentiation and negative value exhibits fractional integration $\left({ }_{c} \mathscr{I}_{t}^{\lambda}\right)$ (Chen et al., 2009; Gutiérrez et al., 2010).

In the literature, multiple definitions are available having similar characteristics with special conditions. In particular, the Riemann-Liouville (R-L) definition is utilized in this work, which is written as

$$
{ }_{c} \mathscr{D}_{t}^{\lambda} x(t)=\frac{1}{\Gamma(n-\lambda)}\left(\frac{d}{d t}\right)^{n} \int_{c}^{t} \frac{x(\tau)}{(t-\tau)^{\lambda+1-n}} d \tau
$$


where $n-1<\lambda<n, n \in N$ and $\Gamma$ denote Euler's gamma function. Laplace transforms of the fractional derivative and integral with zero initial condition are given by

$$
\begin{aligned}
& \mathscr{L}\left[{ }_{0} \mathscr{D}_{t}^{\lambda} x(t)\right]=s^{\lambda} X(s) \\
& \mathscr{L}\left[\mathscr{I}_{0}^{\lambda} x(t)\right]=\frac{1}{s^{\lambda}} X(s)
\end{aligned}
$$

where $s^{\lambda}$ is a fractional Laplacian operator.

\section{Haar wavelet operational matrix of FO integration}

In this work, the orthogonal Haar wavelet functions are utilized due to high precision, mathematical simplicity, noise immunity and ease of implementation with other standard algorithms. Computationally, Haar wavelets are faster than other functions of the wavelet family (Chen and Hsiao, 1999), and can be defined as (Li et al., 2015)

$$
h_{m}(t)=h_{1}\left(2^{i} t-k T_{f}\right)
$$

where $T_{f}$ is the total time period, $m=2^{i}+k$, and $i(i \geq 0), k\left(0 \leqslant k \leqslant 2^{i}\right)$ are integers:

$$
h_{0}(t)=1 \text { for } 0 \leqslant t<T_{f} \text { and } h_{1}(t)=\left\{\begin{array}{lc}
1 & 0 \leqslant t<\frac{T_{f}}{2} \\
-1 & \frac{T_{f}}{2} \leqslant t<T_{f} \\
0 & \text { otherwise }
\end{array}\right.
$$

The orthogonal basis functions such as block pulse, Walsh, Chebyshev, and Haar are capable of converting real order differential equations into simple algebraic equations (Kothari et al., 2018; Li et al., 2015). The operational matrix of FO integration (FOI) or differentiation will not only reduce the computation burden, but also make it possible to identify the system with arbitrary orders. Now, an arbitrary function $f(t) \in L^{2}\left[0, T_{f}\right]$ can be written in terms of Haar wavelets for the first $M$ number of terms as

$$
f(t)=\sum_{i=0}^{M-1} f_{i} h_{i}(t)=F_{M}^{T} H_{M}(t)
$$

Here, $F_{M} \triangleq\left[f_{0}, f_{1}, \ldots, f_{M-1}\right]^{T}$ is the Haar coefficient vector and $H_{M}(t) \triangleq\left[h_{0}(t), h_{1}(t), \ldots, h_{M-1}(t)\right]^{T}$ is the Haar function vector. If collocation points are considered as $t_{i}=(2 i-1) T_{f} / 2 M, i=1,2, . ., M$, the $M$-square Haar matrix $\Omega_{M \times M}$ can be defined by

$$
\Omega_{M \times M} \triangleq\left[H_{M}\left(\frac{1}{2 M} T_{f}\right) H_{M}\left(\frac{3}{2 M} T_{f}\right) \ldots H_{M}\left(\frac{2 M-1}{2 M} T_{f}\right)\right]
$$

The integration of the Haar wavelet can be obtained by its multiplication with $M$-square matrix $P_{M \times M}^{\lambda}$. The algebraic expression of FOI of the Haar wavelet is written as

$$
\left(\mathscr{I}^{\lambda} H_{M}\right)(t) \approx P_{M \times M}^{\lambda} H_{M}(t)
$$

where $M$-square matrix $P_{M \times M}^{\lambda}$ is called the HWOM of FOI, which is computed as given by Li et al. (2015)

$$
P_{M \times M}^{\lambda}=\Omega_{M \times M} F^{\lambda} \Omega_{M \times M}^{-1}
$$

where $F^{\lambda}$ is the $M$-square generalized operational matrix of FOI for block pulse functions (Tang et al., 2017) and can be described as

$$
F^{\lambda}=\left(\frac{T_{f}}{M}\right)^{\lambda} \frac{1}{\Gamma(\lambda+2)}\left(\begin{array}{ccccc}
f_{1} & f_{2} & f_{3} & \ldots & f_{M} \\
0 & f_{1} & f_{2} & \ldots & f_{M-1} \\
\vdots & \ddots & f_{1} & \ldots & f_{M-2} \\
\vdots & & \ddots & \ddots & \vdots \\
0 & \ldots & \ldots & 0 & f_{1}
\end{array}\right)
$$

where $f_{1}=1, f_{q}=q^{\lambda+1}-2(q-1)^{\lambda+1}+(q-2)^{\lambda+1} \quad$ and $q=2,3, \ldots, M$. Similarly, the delayed Haar wavelet function $H_{M}(t-L)$ can be characterized using a delay operational matrix as

$$
H_{M}(t-L)=E_{H} H_{M}(t)
$$

where $L$ is the input time delay and $M$-square matrix $E_{H}$ is the HWOM of delay, which can be given as (Kothari et al., 2018)

$$
E_{H}=\Omega_{M \times M} E \Omega_{M \times M}^{-1}
$$

where $E$ is generalized delay operational matrix (Tang et al., 2017), given by

$$
E=\left[\begin{array}{ccccccc}
0 & \ldots & 0 & 1 & 0 & \ldots & 0 \\
0 & \ldots & 0 & 0 & 1 & \ldots & 0 \\
\vdots & & \vdots & \vdots & \vdots & \ddots & \vdots \\
0 & \ldots & 0 & 0 & 0 & \ldots & 1 \\
0 & \ldots & 0 & 0 & 0 & \ldots & 0 \\
\vdots & & \vdots & \vdots & \vdots & & \vdots \\
0 & \ldots & 0 & 0 & 0 & \ldots & 0
\end{array}\right]_{(M \times M)}
$$

Therefore, performing fractional integration on delayed Haar function $H_{M}(t-L)$ using equations (8) and (11), we obtain

$$
\begin{array}{r}
\left(\mathscr{I}^{\lambda} H_{M}\right)(t-L)=E_{H}\left(\mathscr{I}^{\lambda} H_{M}\right)(t) \\
=E_{H} P_{M \times M}^{\lambda} H_{M}(t)
\end{array}
$$

Therefore, $\left(\mathscr{I}^{\lambda} H_{M}\right)(t-L)$ is obtained simply by matrix multiplication of $H_{M}(t)$ with $P^{\lambda}$ and $E_{H}$.

Now, consider a FO SISO linear time-invariant system having $n$-poles and $m$-zeros with time delay. The differential equation of this system is given as

$$
\sum_{i=0}^{n} a_{i} D^{\lambda_{i}} y(t)=\sum_{j=0}^{m} b_{j} D^{\mu_{j}} u(t-L)
$$

where $\left(a_{i}, b_{j} \in \mathbb{R}^{2}\right),\left(\lambda_{i}, \mu_{j} \in \mathbb{R}+{ }^{2}\right), y(t)$ and $u(t)$ are the output and input of the system, respectively, and $L$ represents the time delay. After expanding, this expression in general form is 


$$
\begin{aligned}
& a_{n} D^{\lambda_{n}} y(t)+a_{n-1} D^{\lambda_{n-1}} y(t)+\cdots+a_{0} D^{\lambda_{0}} y(t) \\
& =b_{m} D^{\mu_{m}} u(t-L)+b_{m-1} D^{\mu_{m-1}} u(t-L) \\
& +\cdots+b_{0} D^{\mu_{0}} u(t-L)
\end{aligned}
$$

Integrating both sides with order $\lambda_{n}$ gives

$$
\begin{aligned}
& a_{n} y(t)+a_{n-1} I^{\lambda_{n}-\lambda_{n-1}} y(t)+\ldots+a_{0} I^{\lambda_{n}-\lambda_{0}} y(t) \\
& =b_{m} I^{\lambda_{n}-\mu_{m}} u(t-L)+b_{m-1} I^{\lambda_{n}-\mu_{m-1}} u(t-L) \\
& +\ldots+b_{0} I^{\lambda_{n}-\mu_{0}} u(t-L)
\end{aligned}
$$

The measured data can be expressed in terms of a Haar wavelet and written as

$$
\begin{gathered}
u(t-L)=U^{T} H_{M}(t-L) \\
y(t)=Y^{T} H_{M}(t)
\end{gathered}
$$

where $Y^{T}=\left[y_{1}, y_{2}, \ldots, y_{M}\right], U^{T}=\left[u_{1}, u_{2}, \ldots, u_{M}\right]$, and superscript $T$ denotes the transpose. Therefore, the integral of input and output terms can be simplified using equations (18), (19), (8) and (11), and one can obtain the following expressions:

$$
I^{\lambda_{n}-\mu_{m-1}} u(t-L)=U^{T} I^{\lambda_{n}-\mu_{m-1}} E_{H}\left(H_{M}(t)\right)=U^{T} E_{H} P_{M \times M}^{\lambda_{n}-\mu_{m-1}} H_{M}(t)
$$

$$
I^{\lambda_{n}-\lambda_{n-1}} y(t)=Y^{T} I^{\lambda_{n}-\lambda_{n-1}}\left(H_{M}(t)\right)=Y^{T} P_{M \times M}^{\lambda_{n}-\lambda_{n-1}} H_{M}(t)
$$

Using these equations, equation (17) can be rewritten as

$$
\begin{aligned}
& Y^{T}\left(a_{n} I+a_{n-1} P_{M \times M}^{\lambda_{n}-\lambda_{n-1}}+\ldots+a_{0} P_{M \times M}^{\lambda_{n}-\lambda_{0}}\right) \\
& =U^{T} E_{H}\left(b_{m} P_{M \times M}^{\lambda_{n}-\mu_{m}}+b_{m-1} P_{M \times M}^{\lambda_{n}-\mu_{m-1}}+\ldots+b_{0} P_{M \times M}^{\lambda_{n}-\mu_{0}}\right)
\end{aligned}
$$

Finally, one can express the output $y(t)$, using equations (19) and (22), in an algebraic form as

$$
y(t)=U^{T} E_{H} N D^{-1} H_{M}(t)
$$

where $D=\left(a_{n} I+a_{n-1} P_{M \times M}^{\lambda_{n}-\lambda_{n-1}}+\ldots+a_{0} P_{M \times M}^{\lambda_{n}-\lambda_{0}}\right), N=\left(b_{m} P_{M \times M}^{\lambda_{n}-\mu_{m}}\right.$ $\left.+b_{m-1} P_{M \times M}^{\lambda_{n}-\mu_{m-1}}+\ldots+b_{0} P_{M \times M}^{\lambda_{n}-\mu_{0}}\right)$ and $I$ denotes the identity matrix. Equation (23) represents the output $y(t)$ for the generalized $n$ th-order transfer function. Suppose the identification requires a low-order transfer function model with one fractional pole, then the output expression can be characterized from equation (23) as

$$
y(t)=U^{T} E_{H}\left(b_{0} P_{M \times M}^{\lambda_{1}}\right)\left(a_{1} I+a_{0} P_{M \times M}^{\lambda_{1}}\right)^{-1} H_{M}(t)
$$

and the transfer function can be written as

$$
G(s)=b_{0} e^{-L s} /\left(a_{1} s^{\lambda_{1}}+a_{0}\right)
$$

Similarly, the output expression for the fractional double-pole model is obtained as

$$
y(t)=U^{T} E_{H}\left(b_{0} P_{M \times M}^{\lambda_{2}}\right)\left(a_{2} I+a_{1} P_{M \times M}^{\lambda_{2}-\lambda_{1}}+a_{0} P_{M \times M}^{\lambda_{2}}\right)^{-1} H_{M}(t)
$$

and the transfer function is

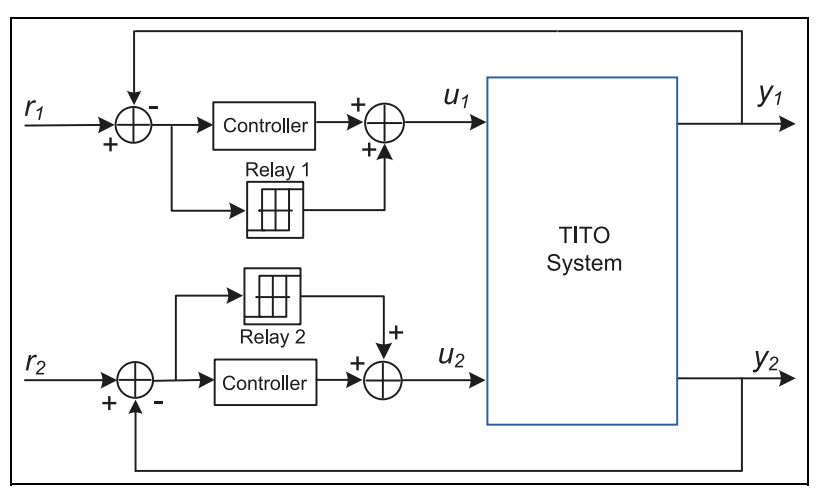

Figure I. Proposed online technique to obtain identification data.

$$
G(s)=b_{0} e^{-L s} /\left(a_{2} s^{\lambda_{2}}+a_{1} s^{\lambda_{1}}+a_{0}\right)
$$

Furthermore, the matrix $P_{M \times M}^{\lambda}$ contains the unknown model parameters and the fractional differential orders. This provides the estimation more simply than performing a complex calculation of fractional differentiation of input and output signals.

\section{Proposed modelling technique for the TITO system}

The aim is to complete the estimation of the TITO system (four transfer function model parameters) from single test run data. The modelling scheme is as shown in Figure 1. During the experiment, the position of two switches changes to collect the online test data. This is different from the conventional relay feedback method where the controller is replaced by the relay. In the identification procedure, a relay height is increased from zero to some acceptable value to induce oscillations (limit cycle) at the output. The present scheme only requires oscillations around the setpoint of the closed-loop system, and no prior knowledge of the controller or system dynamics is required. Furthermore, it is absolutely not necessary to determine the frequency or peak amplitude of the periodic signal. Unlike other procedures in the literature, it is not required to determine the stable frequency, amplitudes and series of test data. Conceptually, the method is a very simple means to obtain the input/output test data of a small period for identification purposes. It is evident that the estimation takes into account the real behaviour of the system under closed-loop conditions. The closed-loop controlled TITO system is represented in terms of the system and controller output signals as

$$
\left[\begin{array}{l}
y_{1} \\
y_{2}
\end{array}\right]=\left[\begin{array}{ll}
g_{11} & g_{12} \\
g_{21} & g_{22}
\end{array}\right]\left[\begin{array}{l}
u_{1} \\
u_{2}
\end{array}\right]
$$

where $\left(u_{1}, u_{2}\right)$ represent inputs and $\left(y_{1}, y_{2}\right)$ represent outputs of the TITO system. Four unknown transfer functions, two for main paths and two for cross-paths, need to be estimated with the help of collected inputs and outputs. As mentioned earlier, the proposed technique is a single-step technique and 


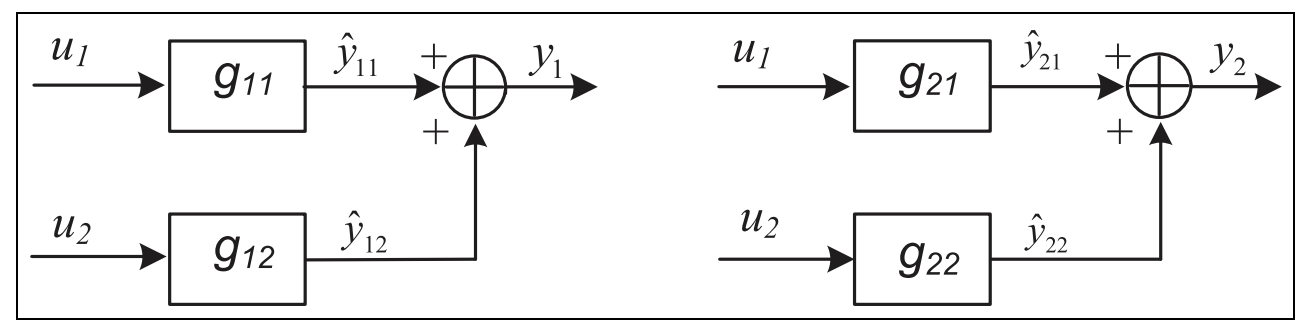

Figure 2. Block diagram of estimated outputs.

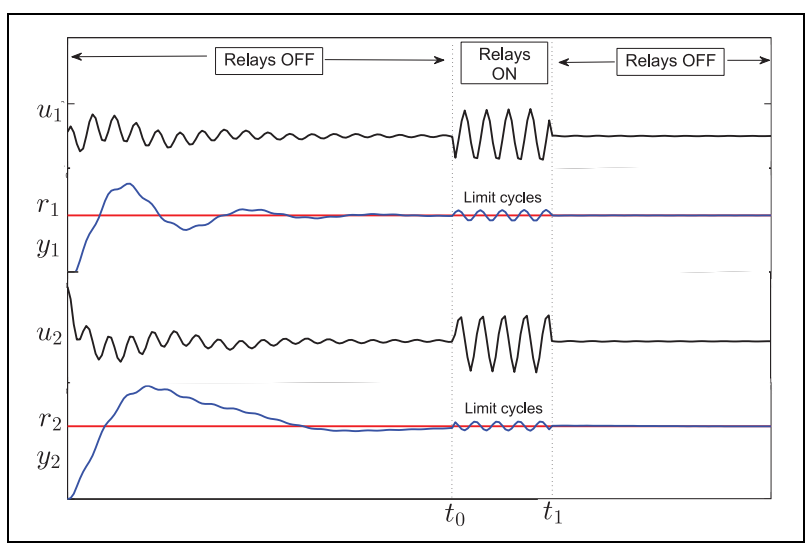

Figure 3. Single-run test data for identification.

accesses only measurable real test data $y_{1}$ and $y_{2}$, for identification purposes. However, it helps to compute immeasurable outputs due to the cross-coupled nature. The estimated output expressions in terms of immeasurable intermediate signals $y_{11}, y_{21}, y_{12}$ and $y_{22}$ can be given as

$$
\begin{aligned}
& y_{1}(t)=\hat{y}_{11}(t)+\hat{y}_{12}(t) \\
& y_{2}(t)=\hat{y}_{21}(t)+\hat{y}_{22}(t)
\end{aligned}
$$

The functional block diagram for estimated outputs is depicted in Figure 2 as per the relation shown in equations (29) and (30).

The first step is to collect appropriate data, and for that switches close at time $t=t_{0}$ to impose relay, parallel to the controller. For the considered setpoints, measurable inputs $\left(u_{1}, u_{2}\right)$ and outputs $\left(y_{1}, y_{2}\right)$ are collected for time up to $t_{1}$, as shown in Figure 3. As per the relay characteristics, the output of the relay is constant until the switching instances. Therefore, the correlation of inputs and outputs is negligible except at the time of relay switching. Due to this reason, even though the test is carried out in a closed loop, the system can be considered as open-loop for identification purposes. Such behaviour of the decentralized relay experiment is presented for the uncoupled TITO system (Berner et al., 2017a). Consider each independent unknown transfer function as $g_{11}, g_{12}, g_{21}$ and $g_{22}$, estimated by the double-pole fractional transfer function:

$$
g_{i j}(s)=\frac{Y_{i j}(s)}{U_{i}(s)}=\frac{b_{0_{i j}}}{a_{2_{i j}} s^{\lambda_{2 i j}}+a_{1_{i j}} s^{\lambda_{1 i j}}+a_{0_{i j}}} e^{-L s}
$$

As given in the previous section, the estimated output of equations (29) and (30) can be rewritten in terms of algebraic equations, using equation (23), as

$$
\begin{aligned}
& y_{1}(t)=U_{1}{ }^{T} E_{H} N_{11} D_{11}^{-1} H_{M}(t)+U_{2}{ }^{T} E_{H} N_{12} D_{12}^{-1} H_{M}(t) \\
& y_{2}(t)=U_{1}{ }^{T} E_{H} N_{21} D_{21}^{-1} H_{M}(t)+U_{2}{ }^{T} E_{H} N_{22} D_{22}^{-1} H_{M}(t)
\end{aligned}
$$

The aforementioned equations show the key contribution to this work. The expressions contain the calculated matrices for all unknown parameters of system transfer functions, and are given by

$$
\begin{aligned}
& N_{11}=b_{0_{11}} P^{\lambda_{211}} \\
& D_{11}=a_{2_{11}} I_{M \times M}+a_{1_{11}} P^{\lambda_{2_{11}}-\lambda_{11}}+a_{0_{11}} P^{\lambda_{211}} \\
& N_{12}=b_{0_{12}} P^{\lambda_{212}} \\
& \left.D_{12}=a_{2_{12}} I_{M \times M}+a_{1_{12}} P^{\lambda_{212}-\lambda_{112}}+a_{0_{12}} P^{\lambda_{212}}\right) \\
& N_{21}=b_{0_{21}} P^{\lambda_{221}} \\
& D_{21}=a_{221} I_{M \times M}+\hat{a}_{1_{21}} P^{\lambda_{221}-\lambda_{1_{21}}}+a_{0_{21}} P^{\lambda_{221}} \\
& N_{22}=b_{0_{22}} P^{\lambda_{222}} \\
& D_{22}=a_{2_{22}} I_{M \times M}+a_{1_{22}} P^{\lambda_{222}-\lambda_{1_{22}}}+a_{0_{22}} P^{\lambda_{222}}
\end{aligned}
$$

Similarly, one can derive expressions for the fractional singlepole model and even generalized $n$-pole and $m$-zero model. Equations (32) and (33) help to estimate two main path transfer functions $\left(g_{11}, g_{22}\right)$ and loop interactions $\left(g_{12}, g_{21}\right)$. Now, in order to identify four transfer functions of the unknown TITO system, the time response data sets, $\left\{u_{1}(k), u_{2}(k), y_{1}(k), y_{2}(k)\right\}_{k=1,2, \cdots M}$ with $M$ samples, are collected after the relay experiment.

At the time of optimization, the performance index, namely the time-moment weighted integral performance criterion, has been used as an objective function to minimize the error. It employs the integral of squared-time-weighted-error (ISTE) to estimate the system parameters, and can be written as

$$
J_{i,(i=1,2)}=\min _{\rho} \sum_{k=1}^{M}\left[k\left(y_{i_{\text {actual }}}(k)-y_{i}(k)\right)\right]^{2}
$$

where $\rho$ is the vector of unknown parameters $\left(b_{0_{i j}}, a_{0_{i j}}, a_{1_{i j}}, a_{2_{i j}}, \lambda_{1_{i j}}, \lambda_{2_{i j}}\right), y_{i}(k)$ is the simulated response, $y_{i_{\text {actual }}}(k)$ is the collected actual data and $M$ denotes the total number of samples. The objective of the optimization is to find the model parameters that would ideally reduce the ISTE as far as possible. The MATLAB function fsolve has been 
Table I. Simple steps of proposed technique for TITO system identification.

\begin{tabular}{ll}
\hline Step I & $\begin{array}{l}\text { Connect two relays parallel to controllers and excite } \\
\text { the considered TITO system. }\end{array}$ \\
Step 2 & $\begin{array}{l}\text { Record the unfiltered data }\left(u_{1}, u_{2}\right) \text { and }\left(y_{1}, y_{2}\right) . \\
\text { Calculate HWOM, } y_{1} \text {, and } y_{2} \text { as per equations }(32) \text { and } \\
\text { Step } 3\end{array}$ \\
Step 4 & $\begin{array}{l}(33) \text { for } M \text { samples. } \\
\text { Use the ISTE objective function (34) to estimate the } \\
\text { optimum parameters. }\end{array}$ \\
\hline
\end{tabular}

ISTE, integral of squared-time-weighted-error; TITO, two-input twooutput.

used in this work to compute the best estimated parameters that satisfy the objective function (equation (34)).

In this analysis, the time-domain error $\varepsilon_{t}$ has been calculated as below for each estimated transfer function parameter and is the basis for the result comparison:

$$
\varepsilon_{t_{i}}=\frac{1}{M} \sum_{k=1}^{M}\left[y_{i}(k)-\hat{y}_{i}(k)\right]^{2}
$$

where $y_{i}(k)$ is the simulated response and $y_{i_{a c t u a l}}(k)$ is the collected actual data from the same input excitation. The frequency-domain identification error $\varepsilon_{f}$ is measured by the worst-case error as

$$
\varepsilon_{f}=\max _{\omega \in\left[0, \omega_{c}\right]}\left\{\left|\frac{\hat{G}(j \omega)-G(j \omega)}{G(j \omega)}\right| \times 100 \%\right\}
$$

where $G(j \omega)$ is the frequency response of the actual model and $\hat{G}(j \omega)$ is the frequency response of the estimated model.

The proposed technique of TITO system identification is explained briefly in Table 1 . In the following section, the technique is verified through numerical examples from the literature and real-time TRMS experimentation.

\section{Results and validation}

The simulation study was conducted, and the algorithm was implemented in the MATLAB environment to identify the well-known Wood and Berry binary distillation column plant and higher-order integer model. In the proposed technique, the selection of datalength value (samples) $M$ is important to achieve the desired accuracy. In the case of Haar wavelets, $M$ is a power of 2 . The study concludes that higher values of $M$ provide better accuracy but reduce the speed of identification. Therefore, the selection of $M$ is a trade-off between speed and accuracy. In general, the desired accuracy can be obtained using $M=256$ with a reasonable speed of the identification routine. Furthermore, the proposed technique has been verified on a real-time TRMS, which is an example of a complicated nonlinear unstable system.

\section{Numerical simulation}

The following examples have been considered for the simulation study:

$$
\begin{gathered}
G_{1}(s)=\left[\begin{array}{cc}
\frac{12.8}{1+16.7 s} e^{-s} & \frac{-18.9}{1+21 s} e^{-3 s} \\
\frac{6.6}{1+10.9 s} e^{-7 s} & \frac{-19.4}{1+14.4 s} e^{-3 s}
\end{array}\right] \\
G_{2}(s)=\left[\begin{array}{ll}
\frac{1}{(s+1)^{2}} e^{-s} & \frac{0.3}{(s+1)^{2}} e^{-s} \\
\frac{0.3}{(s+1)^{2}} e^{-s} & \frac{1}{(0.1 s+1)^{2}} e^{-s}
\end{array}\right]
\end{gathered}
$$

For identification purposes, the single-step data $y_{1}$ and $y_{2}$ have been collected, and corresponding four independent transfer functions for each TITO system have been approximated. In order to collect the data, the relay with height $h_{i}=r_{i} \pm 0.1(i=1,2)$ and proportional gains $K_{p}=1$ were taken, although fairly low values of relay height could be used as per the tolerable plant variable swing. Practically it is only required to generate a small output oscillation for identification. Also, in the case of stable processes, one can use a proportional controller instead of a PID (proportional integral derivative) controller. Table 2 shows the obtained results from the proposed method and the other method by Berner et al. (2017a). The output responses from both actual and estimated models are compared for efficacy purposes. For $G_{1}$, it is clear from Figures 4 and 5 that the technique can handle the noisy signal without pre-processing or filtering the signal, and estimate accurate system dynamics from singlestep test data. The frequency response Nyquist plots show the same agreement in Figure 6. The second example, $G_{2}$, also proves the accuracy in the time domain without noise (Figure 7) and with noise of SNR $=20 \mathrm{~dB}$ (Figure 8). The Nyquist plots are shown in Figure 9 for $G_{2}$.

The above analysis shows that the proposed method identifies parameters accurately and is comparable to another single-step integer-order method (Table 2). The proposed method can deal with noisy signals directly and also useful for any order transfer function models. In the case of FO systems, integer low-order models cannot produce a satisfactory result.

\section{Experimental results on TRMS setup}

Aircraft with rotary wings demonstrate prominent advantages over classical counterparts with fixed wings. In surveillance, small aircraft with rotary wings means only a small space is required for landing and take-off (Toha and Tokhi, 2009). A system like TRMS is the laboratory version of an unmanned helicopter that is specially designed for modelling and controller performance verification. It is rotary wing equipment with significant cross-coupling between its two rotors. The behaviour of this system is similar to that of a flying helicopter, with each rotor influencing angle and position simultaneously. Therefore, for robust and precise control, it demands accurate identification. Sometimes a system model is also useful for investigating behaviour without damaging physical parts.

Some contributions have been shown in the literature based on conventional integer-order modelling for the TRMS setup. The higher integer-order linearized TRMS model was obtained first for helicopter models. Ahmad et al. (2001, 2003) developed a linear identification method for both degree of freedom (2-DOF) and one degree of freedom 
Table 2. Identification results.

\begin{tabular}{|c|c|c|c|c|c|c|}
\hline $\begin{array}{l}\text { Process } \\
G_{l}(s)\end{array}$ & \multirow{2}{*}{ Method } & Identified models & \multicolumn{2}{|l|}{$\varepsilon_{t}$} & \multicolumn{2}{|l|}{$\varepsilon_{f}$} \\
\hline$G_{1}(s)$ & & {$\left[\begin{array}{cc}\frac{12.67}{\mid 7.01 s^{0.952}+0.95} e^{-0.84 s} & \frac{-19.49}{21.01 s^{1.02}+1.02} e^{-2.64 s} \\
\frac{6.63}{\left|0.89 s^{0.89}+0.9\right|} e^{-6.75 s} & \frac{-20.43}{\mid 4.37 s^{0.987}+1.01} e^{-2.74 s}\end{array}\right]$} & {$[0.0268$} & $0.1348]$ & {$\left[\begin{array}{l}4.5087 \\
6.4401\end{array}\right.$} & $\left.\begin{array}{l}7.7185 \\
4.4085\end{array}\right]$ \\
\hline & Proposed $(S N R=20)$ & {$\left[\begin{array}{cc}\frac{12.67}{17.1 s^{0.95}+0.95} \mathrm{e}^{-0.84 s} & \frac{-19.49}{21.0 s^{1.02}+1.02} \mathrm{e}^{-2.64 s} \\
\frac{6.63}{10.88 s^{0.89}+0.90} \mathrm{e}^{-6.74 s} & \frac{-20.43}{14.37 s^{0.98}+1.01} \mathrm{e}^{-2.74 s}\end{array}\right]$} & {$[0.3740$} & $0.4582]$ & {$\left[\begin{array}{l}4.0348 \\
6.9687\end{array}\right.$} & $\left.\begin{array}{l}6.4044 \\
4.9170\end{array}\right]$ \\
\hline & Berner et al. (20I7a) & {$\left[\begin{array}{ll}\frac{15.9}{21.0 s+1} \mathrm{e}^{-1.03 s} & \frac{-18.2}{20.7 s+1} \mathrm{e}^{-3.00 s} \\
\frac{5.84}{9.62 s+1} \mathrm{e}^{-7.02 s} & \frac{-20.2}{15.1 s+1} \mathrm{e}^{-2.99 s}\end{array}\right]$} & {$[1.5146$} & $0.0466]$ & {$\left[\begin{array}{c}12.9246 \\
3.2263\end{array}\right.$} & $\begin{array}{l}7.7301 \\
2.8706\end{array}$ \\
\hline$G_{2}(s)$ & Proposed & {$\left[\begin{array}{cc}\frac{1.107}{2.053 s^{0.904}+1.1} \mathrm{e}^{-1.543 s} & \frac{0.323}{2.166 s^{1.001}+1.006} \mathrm{e}^{-1.443 s} \\
\frac{0.314}{2.178 s^{1.0413}+0.984} \mathrm{e}^{-1.351 \mathrm{~s}} & \frac{0.981}{0.090 s^{1.047}+1.481} \mathrm{e}^{-1.061 \mathrm{~s}}\end{array}\right]$} & {$[0.0907$} & $0.0063]$ & {$\left[\begin{array}{l}8.2123 \\
4.5299\end{array}\right.$} & $\begin{array}{c}7.6140 \\
10.0234\end{array}$ \\
\hline & Proposed $(S N R=20)$ & {$\left[\begin{array}{cc}\frac{1.02}{2.02 s^{1.08}+1.4} \mathrm{e}^{-1.1 s} & \frac{0.316}{2.176 s^{0.98}+0.98} \mathrm{e}^{-1.4 s} \\
\frac{-0.319}{2.216 s^{1.01}+0.95} \mathrm{e}^{-1.4 s} & \frac{0.94}{0.07 s^{0.9}+1.07} \mathrm{e}^{-1.2 s}\end{array}\right]$} & {$[0.1850$} & $0.0155]$ & {$\left[\begin{array}{l}4.0527 \\
7.9155\end{array}\right.$} & $\begin{array}{l}5.7606 \\
17.2017\end{array}$ \\
\hline & Berner et al. (2017a) & {$\left[\begin{array}{ll}\frac{1.13}{2.09 s+1} \mathrm{e}^{-1.43 s} & \frac{0.32}{2.17 s+1} \mathrm{e}^{-1.33 s} \\
\frac{0.32}{1.90 s+1} \mathrm{e}^{-1.47 s} & \frac{1.02}{0.17 s+1} \mathrm{e}^{-1.04 s}\end{array}\right]$} & {$[0.1612$} & $0.0181]$ & {$\left[\begin{array}{l}3.7090 \\
7.9213\end{array}\right.$} & $\left.\begin{array}{l}5.0108 \\
5.3415\end{array}\right]$ \\
\hline
\end{tabular}

SNR, signal-to-noise ratio

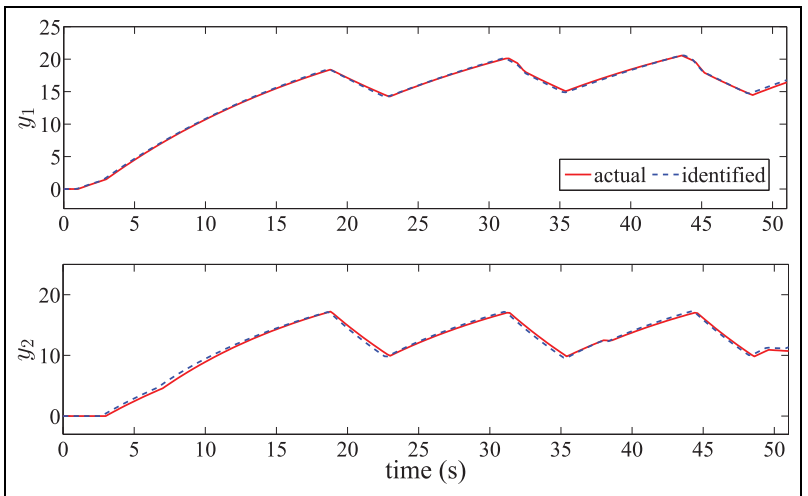

Figure 4. $G_{1}$ : outputs from the actual and identified.

(1-DOF) TRMS systems. The parametric linear model-based technique was proposed using a genetic algorithm by Toha and Tokhi (2009), while the linear time-varying model was approximated using the subspace method (Tanaka et al., 2011). A least square-based parameter identification was explained by Belkheiri et al. (2009). A nonlinear identification method was illustrated by Subudhi and Jena (2009) using metaheuristic optimization algorithms. First-principles modelling has been used for TRMS (Chalupa et al., 2015). A technique based on active disturbance rejection control was developed by Yang et al. (2016), and more recently identification using the Euler-Lagrange technique was described by Tastemirov et al. (2017). This study gives a motivation for further investigation and accurate identification of a highly cross-coupled system with nonlinear and unstable behaviour.

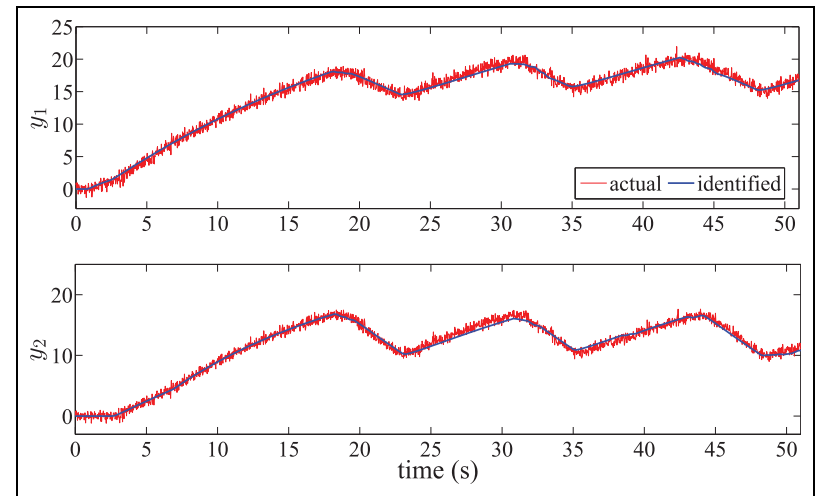

Figure 5. $G_{1}$ : noisy $(S N R=20)$ data and identified model output.

Unlike a helicopter, the TRMS is attached to a tower and the desired pitch and yaw angle can be achieved through rotor speed variation. The experimental setup for TRMS is depicted in Figure 10, with an electrical and a mechanical unit. The mechanical unit is made of a rotary unit attached to a tower. The rotary unit comprises two rotors: the main rotor (pitch) and tail rotor (yaw), positioned on a beam for counterbalance. The electrical unit is responsible for measurable output signal and control signals transfer between the TRMS and computer. The physical parameters of TRMS are described in the lab manual (Feedback, 2008).

Due to multiple inputs, significant cross-coupling can be observed, and system behaviour is nonlinear. The identified model including cross-coupling can be used for control design. Figure 11 shows loop interaction in the TRMS system 


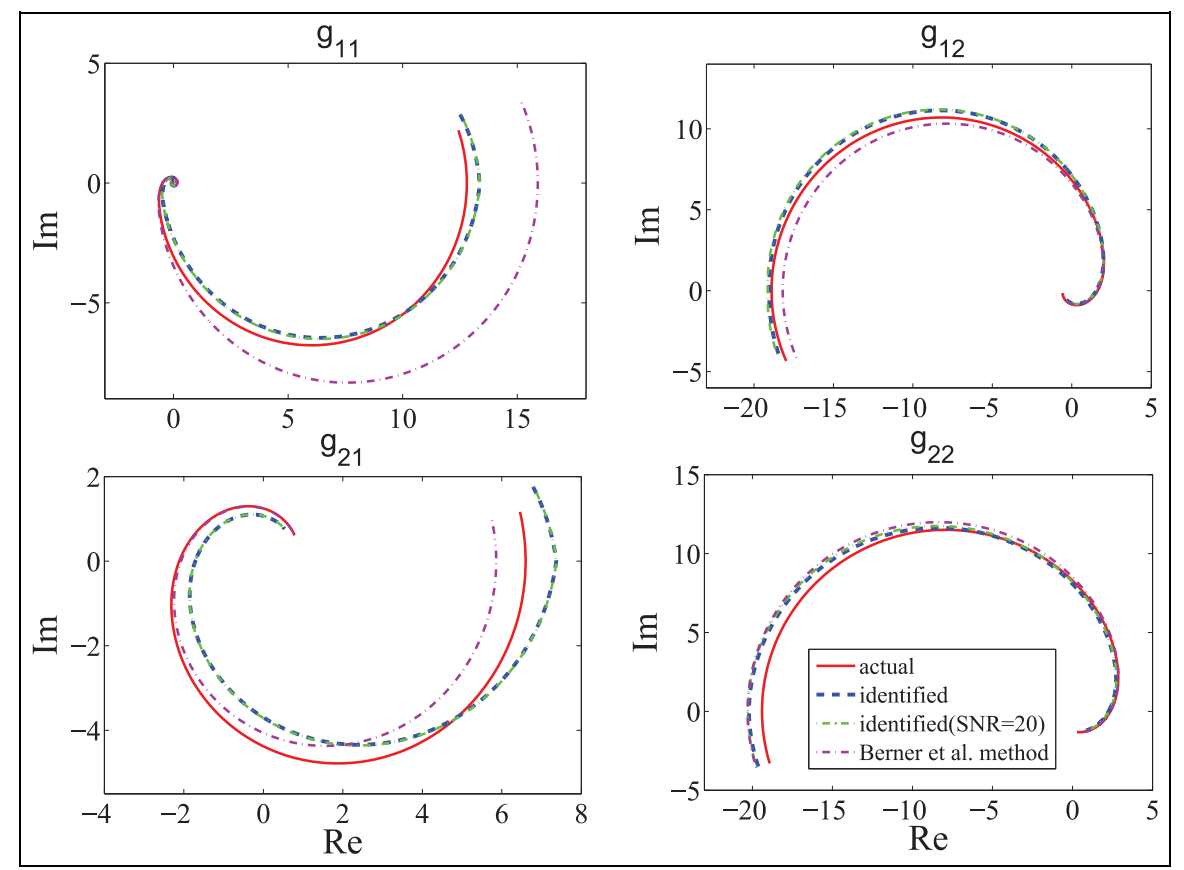

Figure 6. Frequency-domain responses for $G_{1}$.

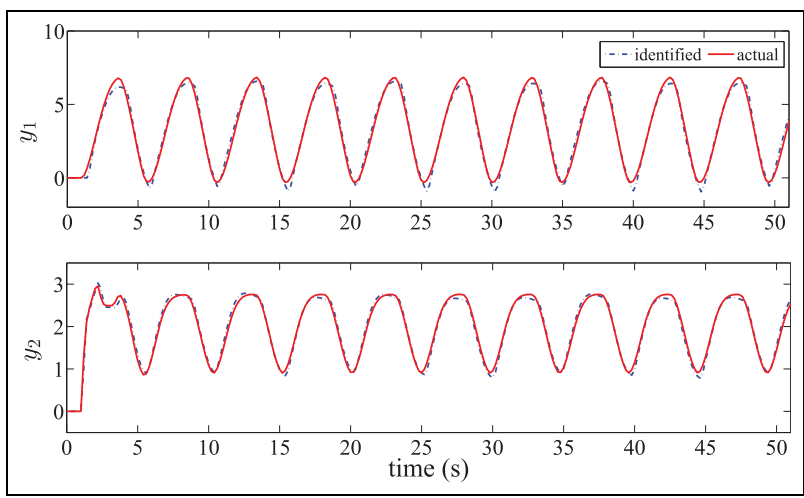

Figure 7. $G_{2}$ : outputs from the actual and identified.

whereby signals $u_{1}, u_{2}$ are the inputs and $y_{1}, y_{2}$ are the outputs of the TRMS. Here, outputs for main paths are measured with the influence of cross-coupling. Inputs are provided by means of voltages, and the speeds of rotors are controlled for desired yaw angle and pitch angle. Outputs are measured in radians.

For the purpose of identification and evaluation of the presented method, the Simulink model was used first with the same parameters as the real TRMS system. In order to obtain a stable response, the closed-loop configuration is preferred. The single-step data of the TRMS was collected, as mentioned in the previous section. After getting steady-state outputs according to setpoints, the switches close and relays play their role at time $t=t_{0}$. For this experiment, the pitch setpoint $=0.4$ and the yaw setpoint $=0.5$ have been considered with initial PID1 parameters as $K_{p}=3, K_{i}=8$ and $K_{d}=10$, and

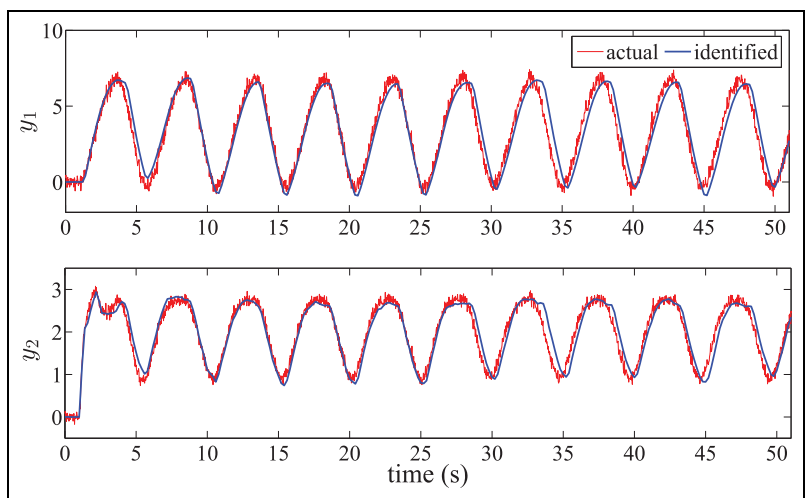

Figure 8. $G_{2}$ : noisy $(S N R=20)$ data and identified model output.

PID2 as $K_{p}=2, K_{i}=0.5$ and $K_{d}=5$. Due to relay properties, the system produced sustained oscillations around steadystate outputs $\left(y_{1}, y_{2}\right)$, as shown in Figure 12. The sampled inputs $\left(u_{1}, u_{2}\right)$ and outputs $\left(y_{1}, y_{2}\right)$ were collected for identification purposes.

After applying the presented technique, the unknown system models are approximated as follows:

$$
\begin{aligned}
g_{11}(s) & =\frac{4.178}{3.082 s^{2.164}+5.576 s^{0.947}+10.622} \\
g_{12}(s) & =\frac{0.293}{0.993 s^{2.022}+0.916 s^{1.332}+0.348} \\
g_{21}(s) & =\frac{0.522}{0.184 s^{2.435}+1.589 s^{1.115}+10.083}
\end{aligned}
$$



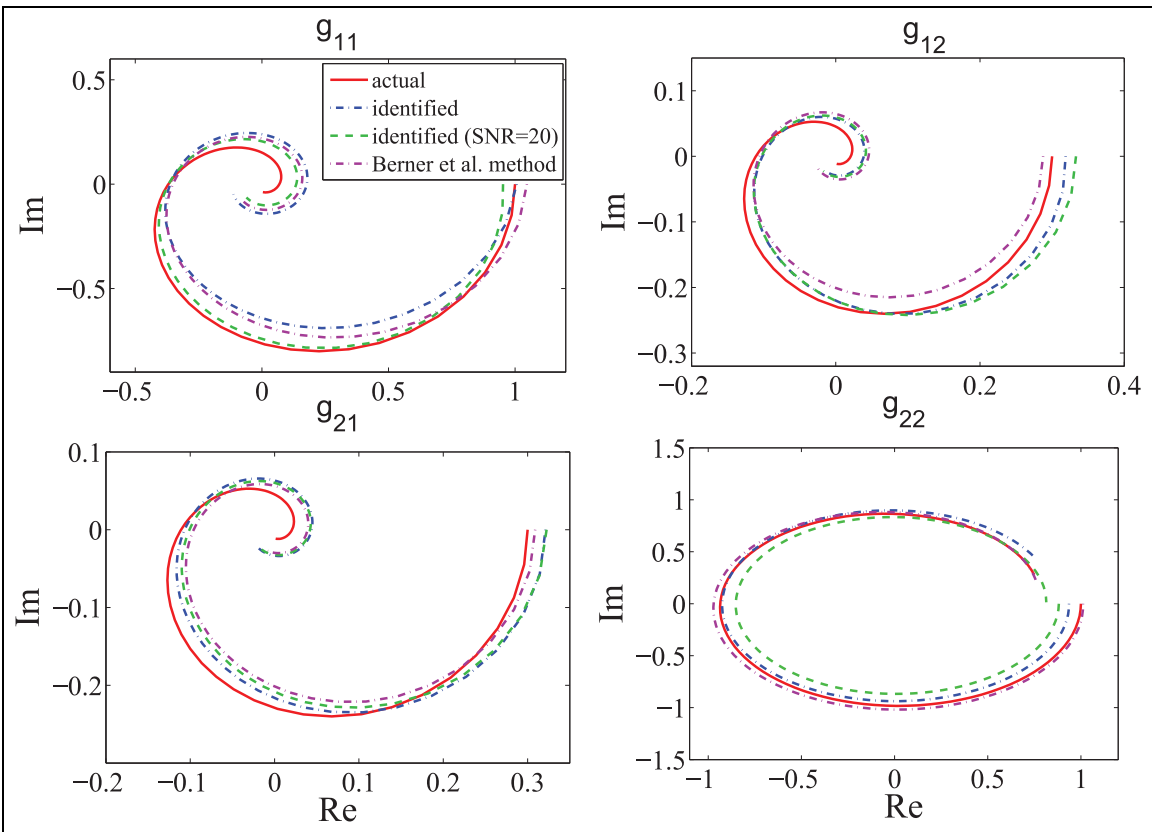

Figure 9. Frequency-domain responses for $G_{2}$.

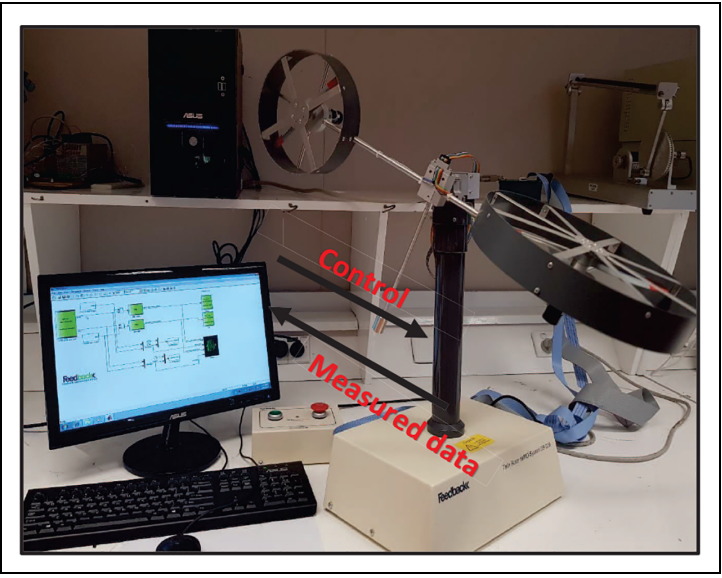

Figure 10. Experimental setup of TRMS.

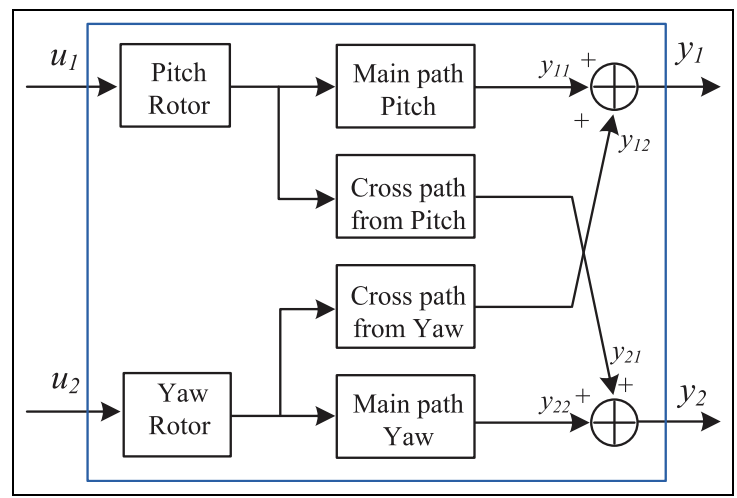

Figure II. Cross-coupling in the twin rotor multiple-input multipleoutput system.

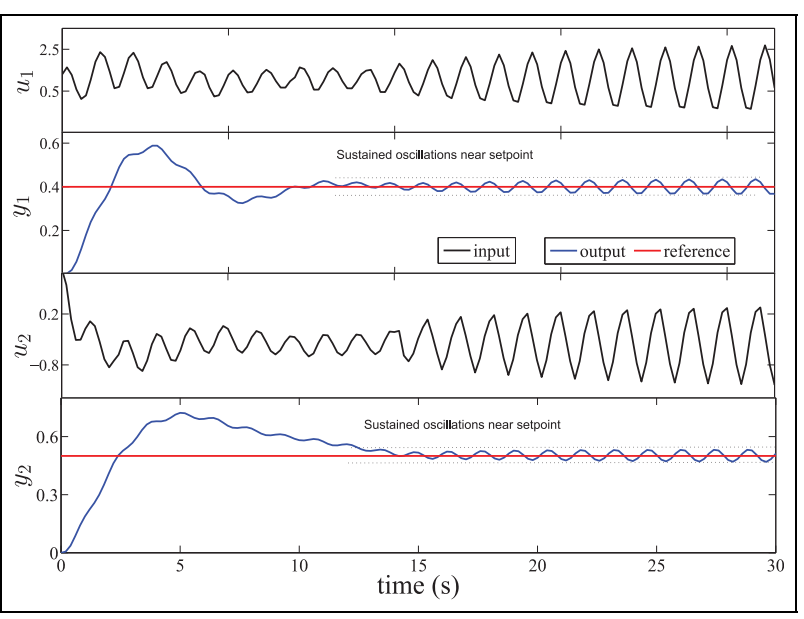

Figure 12. Experimental data for identification.

$$
g_{22}(s)=\frac{9.348}{29.725 s^{1.701}+1.995 s^{1.345}+9.005}
$$

The estimated model performances were verified with respect to actual TRMS outputs. Table 3 shows the verification results with two types of input setpoints. For both step and mixed sinusoidal reference inputs, the identified models depict nearly the same responses as the actual. Figure 13 shows the same agreement with mixed sinusoidal input, and the estimated model output follows the actual output closely.

\section{Conclusions}

In this work, the Haar wavelet-based single-step closed-loop identification strategy for the class of TITO systems is 
Table 3. Verification with various input types.

\begin{tabular}{lllr}
\hline Test & Setpoint type & Pitch and yaw setpoint & Error\% \\
\hline I & Step signal & $r_{1}=0.4$ & $\varepsilon_{t_{1}}=0.76$ \\
& $r_{2}=0.5$ & $\varepsilon_{t_{2}}=0.30$ \\
2 & $r_{1}=0.1 \sin 0.6283 t+0.1 \sin 0.3|4| t+0.1 \sin 0.1256 t+0.4$ & $\varepsilon_{t_{1}}=0.12$ \\
& Random signal (mixed sinusoidal) & $r_{2}=0.8 \sin 0.6283 t+0.3 \sin 0.3|4| t+0.3 \sin 0.06283 t$ & $\varepsilon_{t_{2}}=1.89$ \\
\hline
\end{tabular}

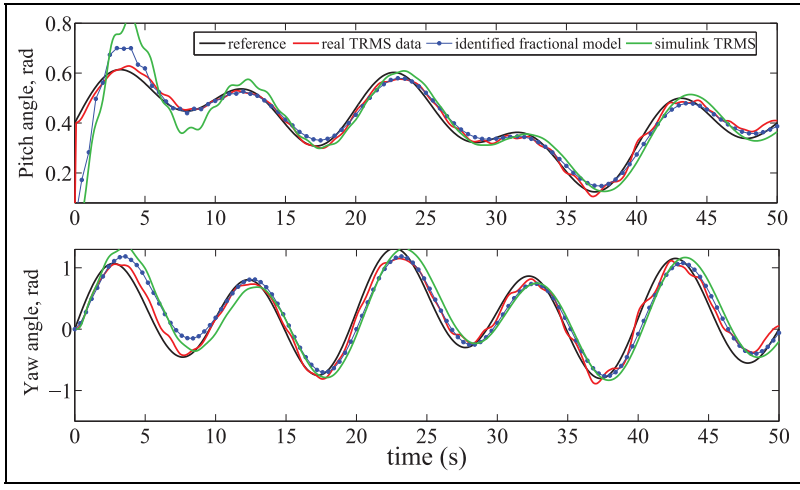

Figure 13. Pitch and yaw subsystems: actual and model outputs.

presented. Even though fractional models are considered, the algebraic operational matrix approach eventually reduces the computational complexity of the identification. The proposed technique can be directly applied on single test run data, and thus could be faster than sequential or two-step approaches. Moreover, the advantage of HWOM-based algebraic matrix multiplication will allow the estimation of all four transfer function parameters with single-step measured data. Concerning the FO model, another advantage of the proposed technique is to have a low-order model that can perform better with fewer unknown parameters. This method can also be applied without controllers in the loop and without non-zero setpoints to collect the identification data. For complex plant like the TRMS, the fractional model represents the system more accurately than the classical counterpart. One downside to this method could be that it is a little less accurate for some class of systems where a two-step or sequential fractional approach can produce a better result than a single-step method. This is because the single-step method has to deal with more unknown parameters concurrently compared to the two-step method.

\section{Declaration of conflicting interests}

The authors declared no potential conflicts of interest with respect to the research, authorship, and/or publication of this article.

\section{Funding}

The authors received no financial support for the research, authorship, and/or publication of this article.

\section{ORCID iD}

Utkal Mehta (iD) https://orcid.org/0000-0001-8613-2490

\section{References}

Ahmad S, Chipperfield A and Tokhi M (2001) Parametric modelling and dynamic characterization of a two-degree-of-freedom twinrotor multi-input multi-output system. Proceedings of the Institution of Mechanical Engineers, Part G: Journal of Aerospace Engineering 215(2): 63-78.

Ahmad SM, Chipperfield AJ and Tokhi MO (2003) Dynamic modelling and linear quadratic Gaussian control of a twin-rotor multiinput multi-output system. Proceedings of the Institution of Mechanical Engineers, Part I: Journal of Systems and Control Engineering 217(3): 203-227.

Bajarangbali and Majhi S (2012) TITO system identification using relay with hysteresis. In: 2012 1st International conference on power and energy in NERIST (ICPEN), Nirjuli, India, 28-29 Dec., pp. $1-5$.

Belkheiri M, Rabhi A, Boudjema F, et al. (2009) Model parameter identification and nonlinear control of a twin rotor MIMO system: TRMS. IFAC Proceedings 42(10): 1487-1492.

Berner J, Soltesz K, Hägglund T, et al. (2017a) Autotuner identification of TITO systems using a single relay feedback experiment. IFAC Papers OnLine 50(1): 5332-5337.

Berner J, Soltesz K, Åström KJ, et al. (2017b) Practical evaluation of a novel multivariable relay autotuner with short and efficient excitation. In: 2017 IEEE conference on control technology and applications (CCTA), Mauna Lani, USA, 27-30 Aug., pp.1505-1510.

Broman H, Lindgren U, Sahlin H, et al. (1999) A TITO system identification approach. Signal Processing 73(1): 169-183.

Chalupa P, Prikryl J and Novak J (2015) Modelling of twin rotor MIMO system. Procedia Engineering 100: 249-258.

Chen CF and Hsiao C (1999) Wavelet approach to optimising dynamic systems. IEE Proceedings: Control Theory and Applications 146(2): 213-219.

Chen Y, Petras I and Xue D (2009). Fractional order control: a tutorial. In: American control Conference, St. Louis, USA, 10-12 June, pp.1397-1411.

Feedback (2008). Feedback instruments: twin rotor MIMO system control experiments. Crowborough: Feedback.

Gutiérrez RE, Rosário JM and Machado JT (2010) Fractional order calculus: basic concepts and engineering applications. Mathematical Problems in Engineering 2010: 375858.

Jin Q, Cheng Z, Dou J, et al. (2012) A novel closed loop identification method and its application of multivariable system. Journal of Process Control 22(1): 132-144.

Kalpana D, Thyagarajan T and Gokulraj N (2015) Modeling and control of non-square MIMO system using relay feedback. ISA Transactions 59: 408-417. 
Kothari K, Mehta U and Vanualailai J (2018) A novel approach of fractional-order time delay system modeling based on Haar wavelet. ISA Transactions 80: 371-380.

Kothari K, Mehta U and Prasad R (2019) Fractional-order system modeling and its applications. Journal of Engineering Science and Technology Review 12(6): 1-10.

Kothari K, Mehta U, Prasad V, et al. (2020) Identification scheme for fractional Hammerstein models with the delayed Haar wavelet. IEEE/CAA Journal of Automatica Sinica 7(3): 882-891.

$\mathrm{Li} \mathrm{Z}$ and Chen Y (2014) Ideal, simplified and inverted decoupling of fractional order TITO processes. IFAC Proceedings 47(3): 2897-2902.

Li S-Y, Cai W-J, Mei H, et al. (2005a) Effective decentralized TITO process identification from closed-loop step responses. Asian Journal of Control 7(2): 154-162.

Li S-Y, Cai W-J, Mei H, et al. (2005b) Robust decentralized parameter identification for two-input two-output process from closed-loop step responses. Control Engineering Practice 13(4): 519-531.

Li Y, Meng X, Zheng B, et al. (2015) Parameter identification of fractional order linear system based on Haar wavelet operational matrix. ISA Transactions 59: 79-84.

Mehta U and Majhi S (2011) On-line identification of cascade control systems based on half limit cycle data. ISA Transactions 50(3): 473-478.

Mehta U and Rojas R (2017) Smith predictor based sliding mode control for a class of unstable processes. Transactions of the Institute of Measurement and Control 39(5): 706-714.

Monje CA, Chen Y, Vinagre BM, et al. (2010) Fractional-order systems and controls. London: Springer.

Nema S and Padhy PK (2015) Identification of two-input two-output process using state-space analysis. IET Control Theory Applications 9(13): 2029-2038.

Pereira RD, Veronesi M, Visioli A, et al. (2017) Implementation and test of a new autotuning method for PID controllers of TITO processes. Control Engineering Practice 58: 171-185.

Petráš I (2011) Fractional-order nonlinear systems: modeling, analysis and simulation. Beijing and Berlin: Higher Education Press and Springer.

Ram VD and Chidambaram M (2014) Closed loop reaction curve method for identification of TITO systems. IFAC Proceedings 47 (1): 989-996
Ram VD and Chidambaram M (2016) Identification of centralised controlled multivariable systems. Indian Chemical Engineer 58(3): 240-254.

Roy P, Das A and Roy BK (2018) Cascaded fractional order sliding mode control for trajectory control of a ball and plate system. Transactions of the Institute of Measurement and Control 40(3): 701-711.

San-Millan A, Feliu-Talegon D, Feliu-Batlle V, et al. (2017) On the modelling and control of a laboratory prototype of a hydraulic canal based on a TITO fractional-order model. Entropy 2017 19(8): 401

Semino D and Scali C (1998) Improved identification and autotuning of PI controllers for MIMO processes by relay techniques. Journal of Process Control 8(3): 219-227.

Subudhi B and Jena D (2009) Nonlinear system identification of a twin rotor MIMO system. In: TENCON 2009: 2009 IEEE region 10 conference, Singapore, 23-26 Jan., pp.1-6.

Tanaka H, Ohta Y and Okimura Y (2011) A local approach to LPVidentification of a twin rotor MIMO system. IFAC Proceedings 44 (1): 7749-7754.

Tang Y, Li N, Liu M, et al. (2017) Identification of fractional-order systems with time delays using block pulse functions. Mechanical Systems and Signal Processing 91: 382-394.

Tastemirov A, Lecchini-Visintini A and Morales-Viviescas RM (2017) Complete dynamic model of the twin rotor MIMO system (TRMS) with experimental validation. Control Engineering Practice 66: 89-98.

Toha SF and Tokhi MO (2009) Real-coded genetic algorithm for parametric modelling of a TRMS. In: 2009 IEEE congress on evolutionary computation, Trondheim, Norway, 18-21 May, pp.2022-2028.

Viswanathan PK, Toh WK and Rangaiah GP (2001) Closed-loop identification of TITO processes using time-domain curve fitting and genetic algorithms. Industrial \& Engineering Chemistry Research 40(13): 2818-2826.

Wang Q-G, Huang B and Guo X (2000) Auto-tuning of TITO decoupling controllers from step tests. ISA Transactions 39(4): 407-418.

Yang X, Cui J, Lao D, et al. (2016) Input shaping enhanced active disturbance rejection control for a twin rotor multi-input multioutput system (TRMS). ISA Transactions 62: 287-298. 